\title{
Hyperbolic metamaterials-based plasmonic biosensor for fluid biopsy with single molecule sensitivity
}

\author{
Kandammathe Valiyaveedu Sreekanth ${ }^{1}$, Mohamed ElKabbash ${ }^{1}$, Yunus Alapan ${ }^{2}$, Efe Ilker Ilker ${ }^{1}$, \\ Michael Hinczewski ${ }^{1}$, Umut A. Gurkan ${ }^{2,3,4,5}$, and Giuseppe Strangi ${ }^{1,6,7,8, *}$ \\ 1 Department of Physics, Case Western Reserve University, 10600 Euclid Avenue, Cleveland, OH 44106, USA \\ 2 Case Biomanufacturing and Microfabrication Laboratory, Mechanical and Aerospace Engineering Department, \\ Case Western Reserve University, Cleveland, OH 44106, USA \\ 3 Biomedical Engineering Department, Case Western Reserve University, Cleveland, OH 44106, USA \\ 4 Department of Orthopedics, Case Western Reserve University, Cleveland, OH 44106, USA \\ 5 Advanced Platform Technology Center, Louis Stokes Cleveland Veterans Affairs Medical Center, Cleveland, OH 44106, USA \\ 6 CNR-NANOTEC Istituto di Nanotecnologia and Department of Physics, University of Calabria, 87036 Rende, Italy \\ 7 Case Comprehensive Cancer Center, Case Western Reserve University, Cleveland, OH 44106, USA \\ 8 IIT, Italian Institute of Technology, Via Morego, 30, 16163 Genova, Italy
}

Received 13 September 2016 / Accepted 31 October 2016

\begin{abstract}
Plasmonic sensors are one of the most powerful optical biosensors, which can be used for the detection of small numbers of molecules at ultra-low concentrations. However, the detection of dilute analytes of low molecular weight $(<500 \mathrm{Da})$ is still a challenging task using currently available plasmonic biosensors. Here, we demonstrate the detection of both lower and heavier molecular weight biomolecules close to single molecule level using a novel plasmonic biosensor platform based on hyperbolic metamaterials. We show experimentally the extraordinary spectral and angular sensitivities of the biosensor platform, from visible to near infrared (NIR) wavelengths, by exciting the bulk plasmon polaritons associated with hyperbolic metamaterials.
\end{abstract}

Key words: Plasmonics, Biosensors, Optical nanosensors, Metamaterials.

\section{Introduction}

A biosensor is an analytical device that detects bioanalytes. It consists of two elements, a sensitive biological element or a bioreceptor that interacts with a specific bioanalyte providing specific detection and a transducer element that translates such interaction into a measurable signal. The transducer element can be electrochemical, piezoelectric, electronic, optical, etc. Optical biosensors are refractometric sensing devices that are capable of measuring changes in the refractive index that take place in the field of an electromagnetic wave supported by the optical structure of the sensor [1]. Surface plasmon resonance (SPR) sensors are one of the most powerful optical biosensors [2-6]. Surface plasmons (SPs) are coherent free electron oscillations that exist at the interface between any two materials where the real part of the dielectric function changes signs across the interface. SPs can be localized surface plasmons (LSPs), or propagating surface plasmons. LSPs are excited in

*e-mail: giuseppe.strangi@case.edu metals with light oscillating near their plasma frequency, which can be strongly tuned by the surrounding medium and particle's shape, if the metal's size is comparable to the resonant wavelength. The propagating surface plasmon, however, is an electromagnetic wave that involves charge motion in the metal and a polarizing electromagnetic wave in the dielectric (polariton) and are commonly referred to as surface plasmon polaritons (SPPs). SPPs are not resonantly excited and their excitation requires the fulfilling of momentum matching conditions via optical elements able to increase the light momentum. The charge oscillation creates a field inside the metal and the dielectric for both LSPs and SPPs. Accordingly, the resonance or momentum matching conditions are very sensitive to any change in the dielectric constant of the surrounding dielectric, resulting in a shift in the resonant/ matched wavelength or angle (in the case of SPPs) that can be measured in terms of the total shift per refractive index unit (RIU) to indicate the sensitivity of the optical transducer. Furthermore, the high confinement of the electromagnetic field associated with SPs means that they can only sense changes in 

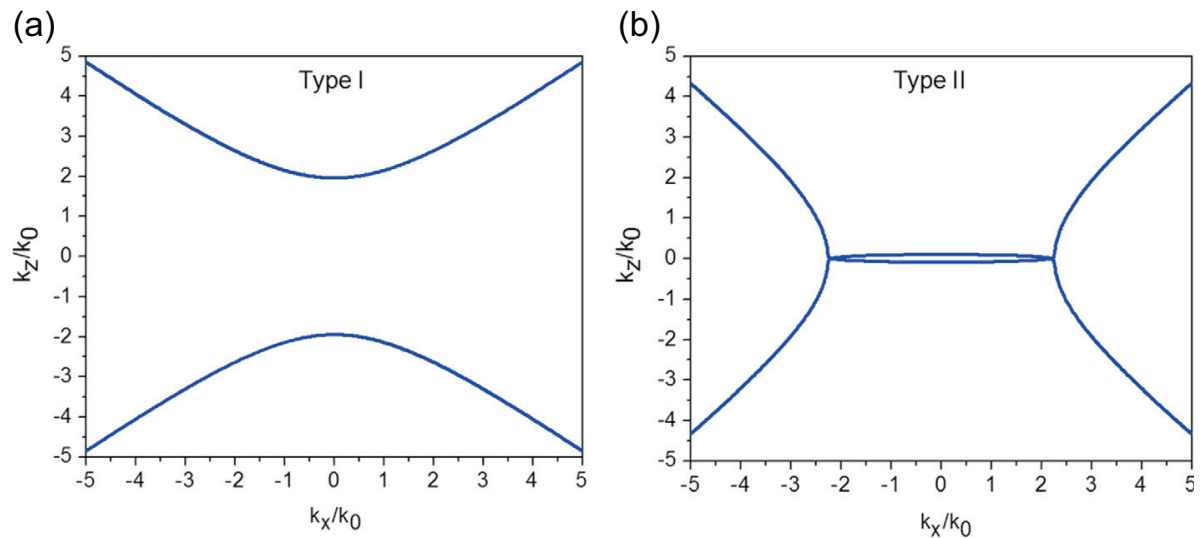

Figure 1. Iso-frequency diagram of (a) type I HMM and (b) type II HMM.

the dielectric constant that take place near their interface, allowing them to "see" only biomolecules that are captured by the bio-receptors. SPP sensors are usually built in a Kretschmann configuration that matches momentum between the surface plasmon and a laser beam, whereas LSP sensors exhibit a tunable resonant frequency that does not require momentum matching [7, 8]. LSP sensors geometrically confine electromagnetic energy absorbed from large optical crosssections to significantly enhance local fields within $5-15 \mathrm{~nm}$ of the nanoparticle surface [9-11]. Although SP biosensors have proven to be successful and are commercialized, they still suffer from several issues: (i) for SPPs, using momentum coupling via the Kretschmann configuration requires bulky optical elements, a prism, which makes it not suitable for point of care (POC) applications. On the other hand, although grating coupled SPP sensors avoid this issue, their sensitivity is an order of magnitude lower than prism coupled SPPs; (ii) while SPs represent the transducer component of the biosensor, one major issue of bio-receptors is the existence of parasitic molecules that cannot be easily distinguished from the targeted biomolecule and result in a false positive signal; (iii) although SP sensors can detect highly diluted analytes, the sensitivity of these sensors are not high enough to detect dilute analytes of low molecular weights $(<500 \mathrm{Da})$. If the sensitivity of SP sensors is increased, POC clinical evaluation, early cancer screening and real time diagnosis of diseases will be possible.

To address these issues, a new sensing platform was needed. Recently, extreme sensitivity has been realized using hyperbolic metamaterials (HMMs) [12-14]. HMMs are a class of artificial materials that show hyperbolic dispersion (Figure 1) because one of their principal permittivity components has the opposite sign to the other two [15]. An interesting consequence of hyperbolic dispersion is that these materials can support waves propagating inside them with infinitely large momentum in the effective medium limit, while such waves are evanescent and decay away exponentially in the superstrate [16]. In our work, we used a type II HMM that consists of a multi-stack of metal/dielectric bilayers. Each bilayer supports a propagating SP wave coupled evanescently to its adjacent bilayer which in turn supports a propagating surface polariton, allowing propagation inside the material as well as on the interface of each bilayer. We call this wave a bulk plasmon polariton (BPP) indicating that it is propagating inside the bulk of the material, while maintain the properties of a propagating surface wave that polarizes its dielectric superstrate. Such BPPs support extremely high momentum and have been shown experimentally to be extremely sensitive to any change in the dielectric constant within the range of its evanescently decaying field in the superstrate. We have demonstrated the excitation of these high $-k$ modes by a grating coupling method [17-19]. However, as we show in the following sections, grating coupling did not affect the high sensitivity of the HMM biosensor. Furthermore, we have shown that exciting BPPs by a grating coupler results in multiple modes that satisfy the momentum matching condition. We show that these modes have different sensitivity for bioanalytes with different molecular weights and thus we provide an extra step towards transducer based specificity.

\section{Sensor development}

\subsection{Design, fabrication, and characterization}

The proposed plasmonic biosensor platform, as illustrated in Figure 2a, is a combination of a metallic diffraction grating and an HMM. Effective medium theory (EMT) [20] was used to design the HMM since the thickness of the individual metal and dielectric layers of the HMM are far below the excitation wavelength. In order to obtain the hyperbolic dispersion in the optical frequencies, 16 alternating thin films of gold and aluminum dioxide $\left(\mathrm{Al}_{2} \mathrm{O}_{3}\right)$ with thickness $16 \mathrm{~nm}$ and $30 \mathrm{~nm}$ respectively were used to fabricate the HMM. EMT-derived uniaxial permittivity components are shown in Figure 2b, which confirms that the fabricated gold- $\mathrm{Al}_{2} \mathrm{O}_{3}$ multilayer is a Type II HMM with hyperbolic dispersion at $\lambda \geq 520 \mathrm{~nm}$, where $\varepsilon_{\|}<0$ and $\varepsilon_{\perp}>0$. In the calculation, experimentally determined optical constants and thickness of both grown thin films were used. In order to excite the modes associated with the HMM, a 2D metallic diffraction grating coupled-HMM 
(a)

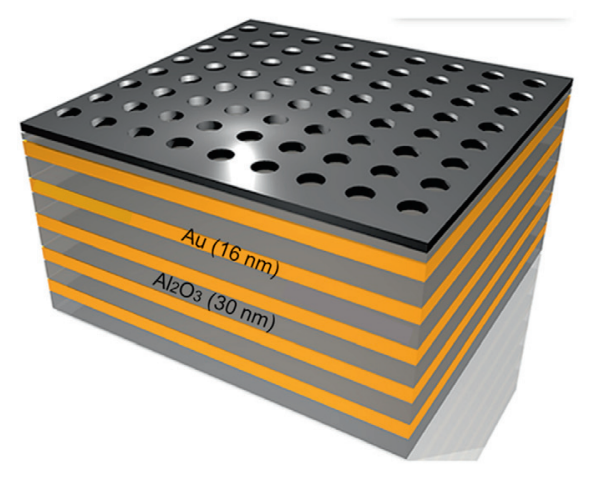

(b)

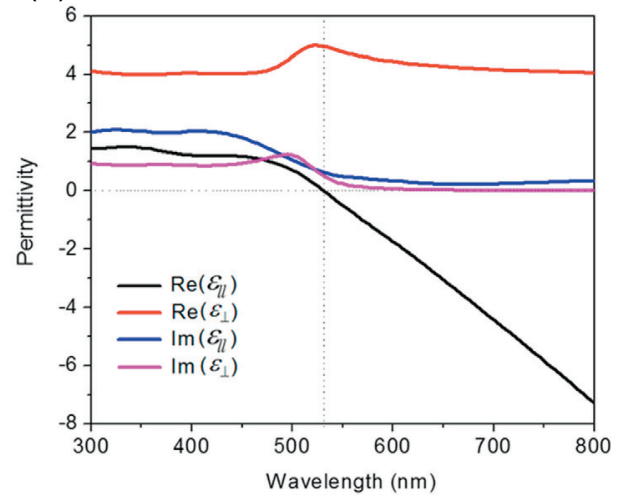

Figure 2. (a) A schematic representation of the fabricated miniaturized diffraction grating coupled HMM sensor device, (b) uniaxial permittivity values (both real and imaginary parts) of $\mathrm{Au}-\mathrm{Al}_{2} \mathrm{O}_{3} \mathrm{HMM}$, determined using effective media theory.

(a)

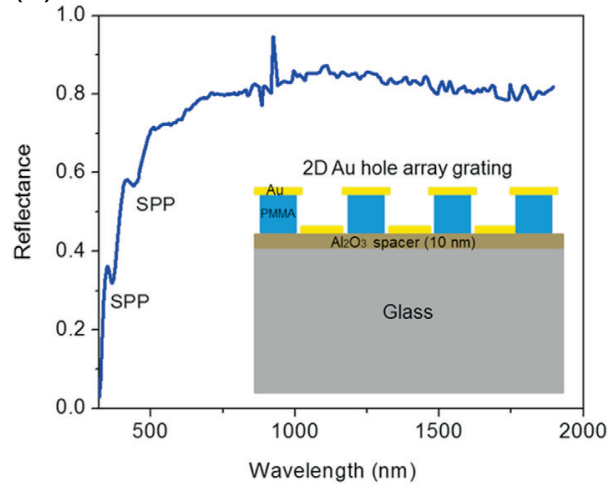

(b)

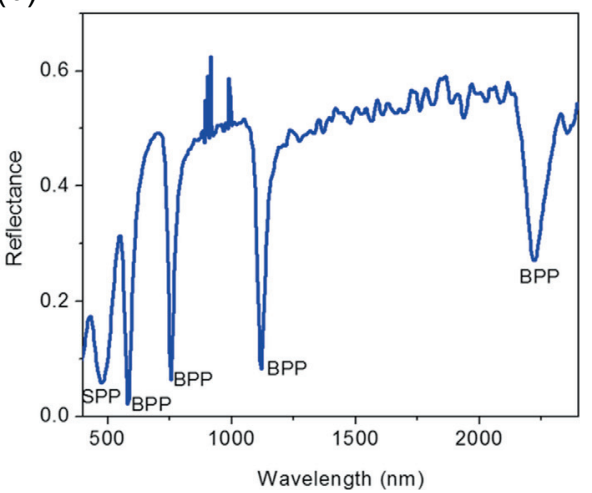

Figure 3. Reflectance spectrum of (a) reference sample and (b) HMM. Angle of incidence is set to $30^{\circ}$.

was designed and fabricated [13]. In this process, a thin $\mathrm{Al}_{2} \mathrm{O}_{3}$ spacer layer with thickness $10 \mathrm{~nm}$ was first deposited on the HMM to improve the coupling between the diffraction grating and the HMM. An array of subwavelength holes with an average period of $500 \mathrm{~nm}$ and hole diameter of $160 \mathrm{~nm}$ was patterned on top of the spacer layer using electron-beam lithography. In order to make a gold diffraction grating, especially for biosensing, a thin gold layer of $20 \mathrm{~nm}$ thickness was directly deposited on top of the sample. As described above, the grating coupling technique was adapted to excite plasmon modes associated with the HMM. In accordance with this coupling technique, the surface plasmon modes can be excited when the wavevectors of the grating diffraction orders are greater than the incident light one. Multiple modes are possible for a given grating period that satisfy the momentum matching condition to bulk plasmon polaritons. Once this takes place, energy is transferred to these high- $k$ modes. The momentum matching condition is given by, $k_{\mathrm{spp}}^{2}=n_{0}^{2} k_{0}^{2} \sin ^{2} \theta \pm 2 n_{0} m k_{\mathrm{g}} k_{0} \sin \theta \cos \phi+\left(m k_{\mathrm{g}}\right)^{2}$, where $\theta$ is the incident grazing angle, $\phi$ is the azimuthal angle, $n_{0}$ is the refractive index of incident medium, $k_{0}=2 \pi / \lambda$ is the vacuum wavevector, $m$ is the grating diffraction order and $k_{\mathrm{g}}=2 \pi / \Lambda$ is the grating wavevector, with $\Lambda$ the grating period. When $\phi=0, k_{\mathrm{spp}}=n_{0} k_{0}$ sin $\theta \pm m k_{\mathrm{g}}$. In our experiments, the polarization of the beam is set to $p$-polarization (TM) and $\phi=0$.

\subsection{Excitation of high q-factor bulk plasmon modes}

To determine the existence of bulk plasmon modes in the fabricated HMM, we acquired the reflectance spectra of the HMM as a function of excitation wavelength and incident angle using a variable angle high-resolution spectroscopic ellipsometer. The spectroscopic and angular resolution of the ellipsometer are $0.03 \mathrm{~nm}$ and $0.01^{\circ}$, respectively. Since the bulk plasmon modes represent the entire family of gap plasmon modes of a multilayer, large modal indices are possible. This corresponds to strong mode confinement and shorter propagation length, which in turn provide high quality $(Q)$ factor modes [17]. We studied the properties of BPP modes by recording the reflectance spectra as a function of excitation wavelength at a particular incident angle (Figure 3b). In contrast to a reference sample (Figure 3a), the HMM shows four high $Q$-factor modes above $500 \mathrm{~nm}$ wavelength. These deepest 
(a)

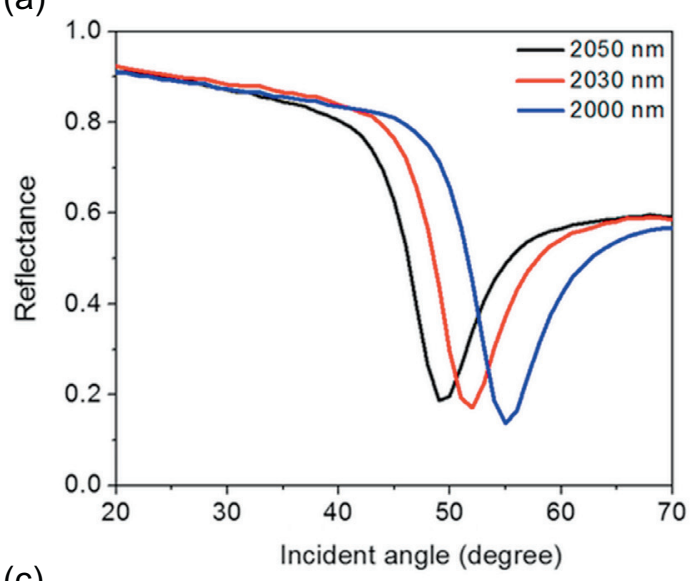

(c)

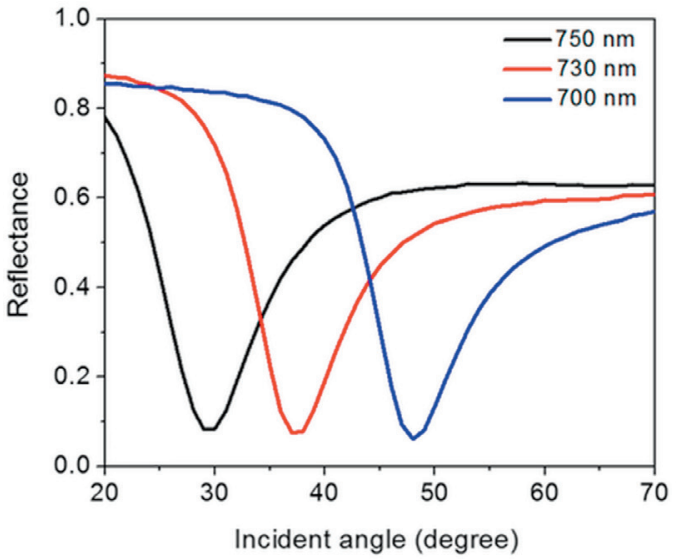

(b)

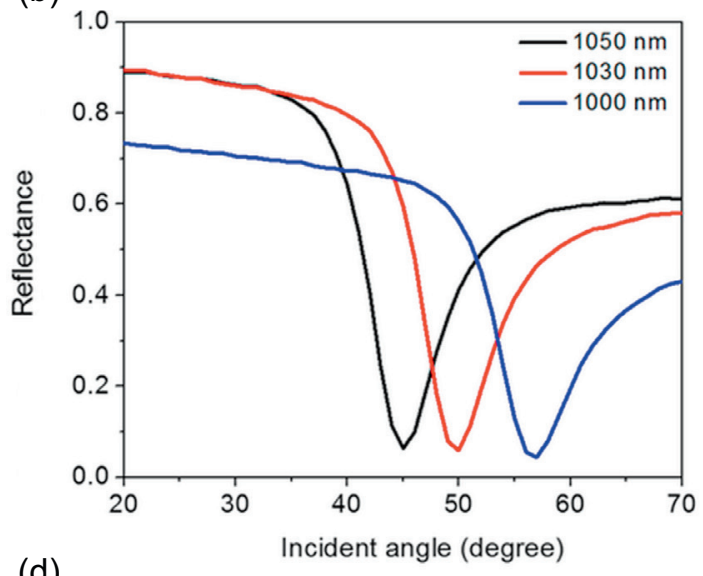

(d)

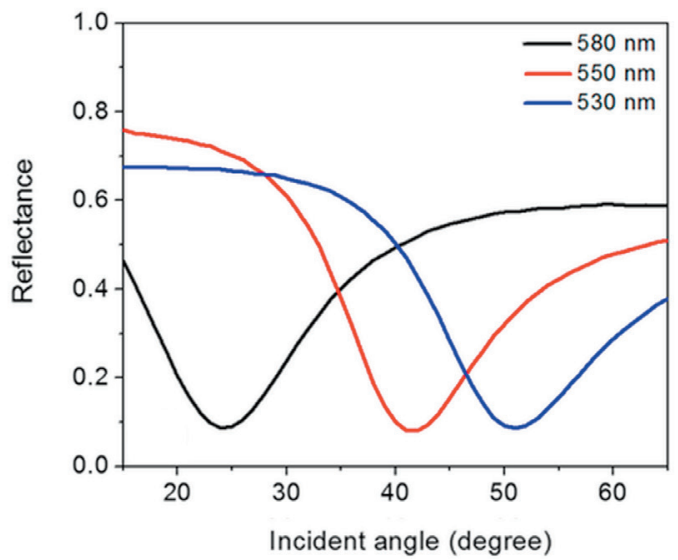

Figure 4. Excitation of BPP modes at each BPP mode band. Reflectance spectra as a function of incident angle: (a) 2050-2000 nm, (b) 1050-1000 nm, (c) 750-700 nm, and (d) 580-530 nm.

narrow reflectance minima represent the existence of highly confined BPP modes in the HMM. The calculated $Q$-factor of modes are 29.5, 26, and 23 at $1120 \mathrm{~nm}, 755 \mathrm{~nm}$, and $580 \mathrm{~nm}$, respectively. That means $Q$-factor increases with the increase in excitation wavelength. The mode at longest wavelength provides the maximum $Q$-factor and the mode at shortest wavelength provides the minimum $Q$-factor, which is useful for the design and development of multi-analyte biosensors. However, the mode at $2200 \mathrm{~nm}$ provides a lower $Q$-factor than these three modes and this mode is not present in the reflectance spectrum when the HMM is integrated with a microfluidic channel because of the greater absorption capacity of the PMMA channel, so the BPP mode at $2200 \mathrm{~nm}$ is not further considered for biosensing. Since BPP mode excitation depends on incident angle, we measured the reflectance spectra of the HMM as a function of incident angle, by fixing an excitation wavelength from the BPP mode bands (Figure 3b). The excitation of BPP modes at different excitation wavelengths in each BPP mode band are shown in Figure 4. A decrease in coupling angle variation with a maximum for the shorter wavelength band and a minimum for the longer wavelength band are observed when the excitation wavelength in each BPP mode band is increased [21].

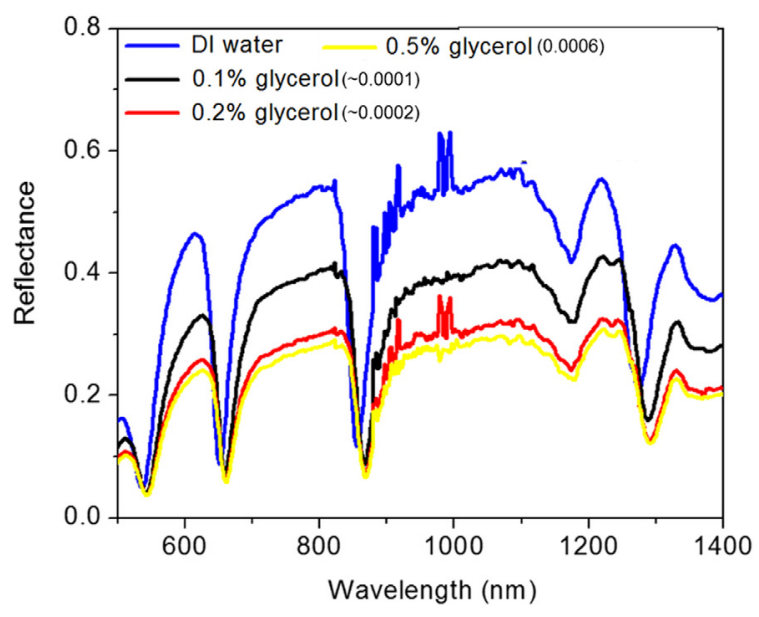

Figure 5. Standard sensor calibration test in spectral interrogation scheme, by injecting different weight percentage concentration of glycerol in distilled water $(0.1-0.5 \% \mathrm{w} / \mathrm{v})$. Reflectance spectra of sensor device with different weight percentage of glycerol. Angle of incidence is set to $30^{\circ}$. The refractive index difference between DI water and the level of glycerol concentration evaluated is shown in the legend. 
(a)

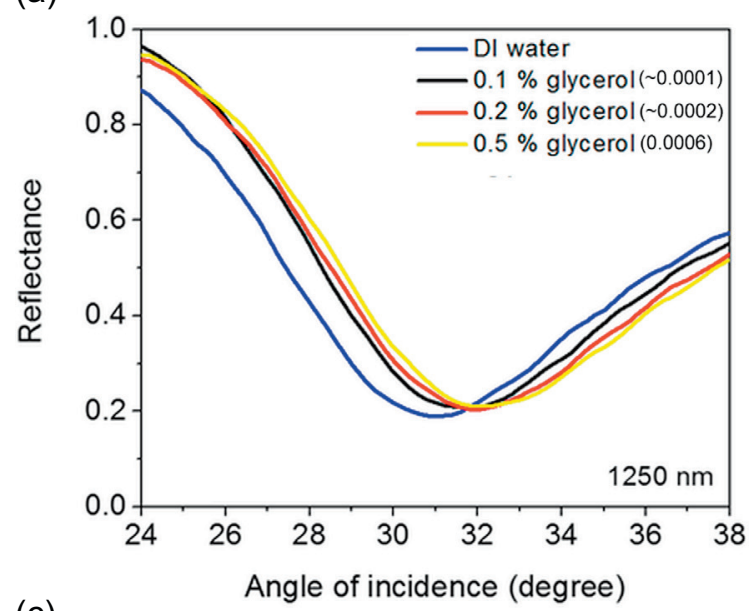

(c)

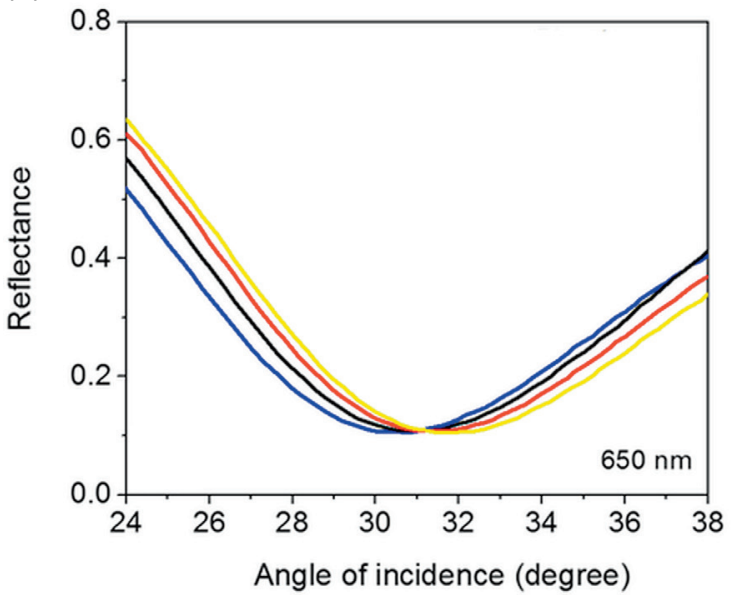

(b)
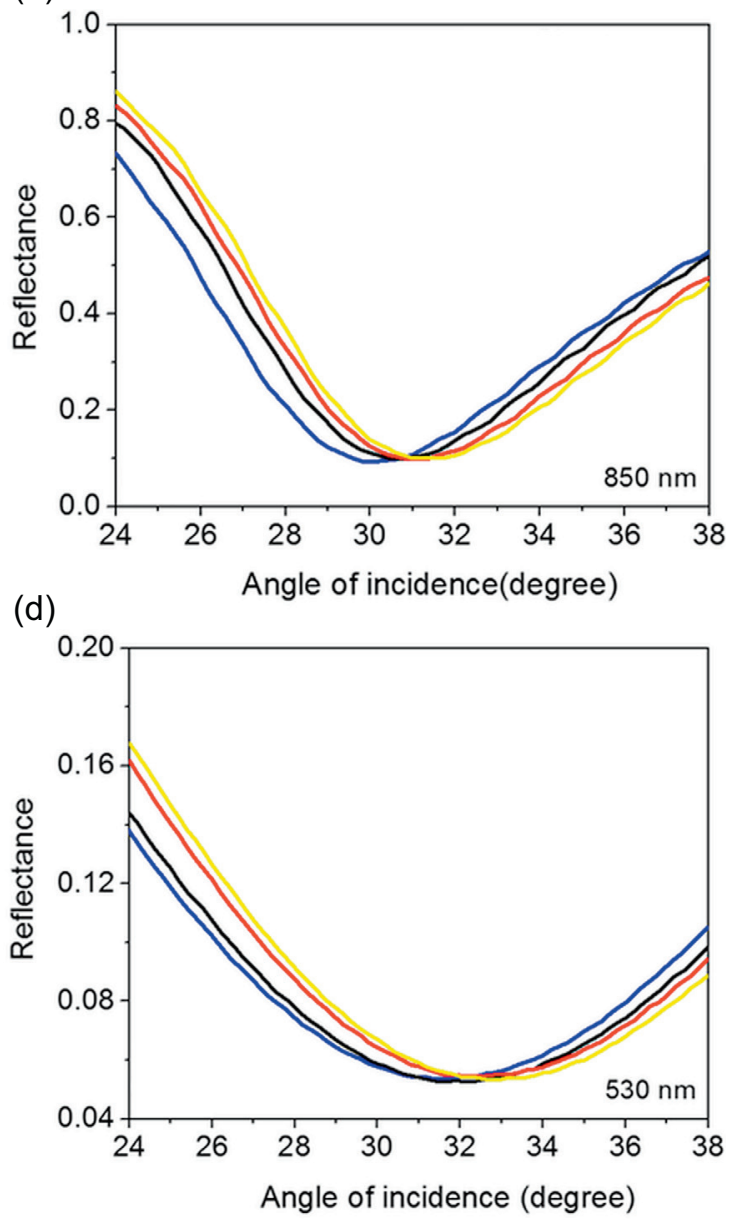

Figure 6. Standard sensor calibration test in angular interrogation scheme. Reflectance spectra as a function of incident angle of the sensor device obtained by injecting different weight percentage concentration of glycerol in distilled water $(0.1-0.5 \% \mathrm{w} / \mathrm{v})$ : (a) at $1250 \mathrm{~nm}$, (b) at $850 \mathrm{~nm}$, (c) $650 \mathrm{~nm}$, and (d) $530 \mathrm{~nm}$. The refractive index difference between DI water and the level of glycerol concentration evaluated is shown in the legend.

\subsection{Measurement of bulk refractive index changes}

From the above analysis, it is clear that BPP modes show different spectral and angular features in each BPP mode band. Therefore, they could be used to design a potential plasmonic biosensor with different spectral and angular sensitivities for each BPP mode band. Thus, we fabricated a microfluidic channel integrated HMM based miniaturized biosensor platform [13]. This sensor works based on the coupling condition between grating surface modes and BPP modes, allowing one to observe a change in resonance wavelength and resonance angle as the refractive index of the surrounding medium changes.

As a first step, the sensor device was calibrated and we determined the detection limit of the sensor in both spectral and angular scan interrogation schemes, by injecting aqueous solutions of glycerol with different weight ratios into the sensor flow microchannel (sample volume $14 \times 2 \times 0.05 \mathrm{~mm}^{3}$ ). The corresponding extremely small bulk refractive index change was recorded as a wavelength and angular shift in the reflectance spectrum. The reflectance spectra of the sensor with different weight ratios of glycerol in distilled water $(0.1-0.5 \%$ $\mathrm{w} / \mathrm{v})$ for spectral and angular scan interrogation schemes are shown in Figures 5 and 6, respectively. As shown in Figure 5, a red shift in resonance wavelength occurred and the quality factor of the resonance declined when the weight ratio of glycerol concentration increased. Also, a positive angular shift for all four wavelengths was obtained when the glycerol weight ratio was increased (Figure 6). A considerable shift even in the case of $0.1 \%(\mathrm{w} / \mathrm{v})$ glycerol indicates the ability of the sensor to detect extremely small refractive index changes. It should be noted that both spectral and angular shift varied between the different modes since the transverse decay of the field in the superstrate strongly varies from one mode to another [13]. This nonlinear variation of shift with glycerol concentration indicates that the sensor has its highest performance at the lowest concentrations. In order to determine the spectral and angular detection limit of the sensor device, we have calculated the spectral and angular sensitivity of the device at each BPP mode band by considering the shift obtained at $0.5 \%(\mathrm{w} / \mathrm{v})$ glycerol. The refractive index difference between DI water and $0.5 \%(\mathrm{w} / \mathrm{v})$ glycerol in DI water 
(a)

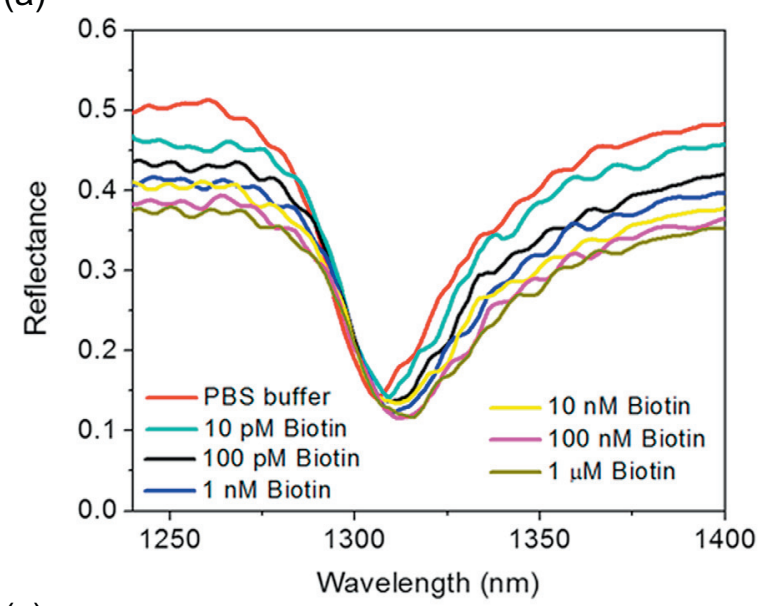

(c)

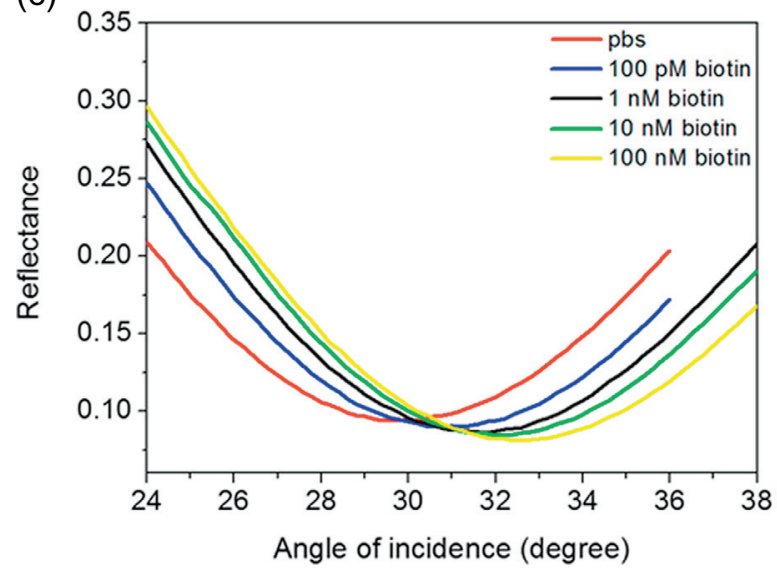

(b)

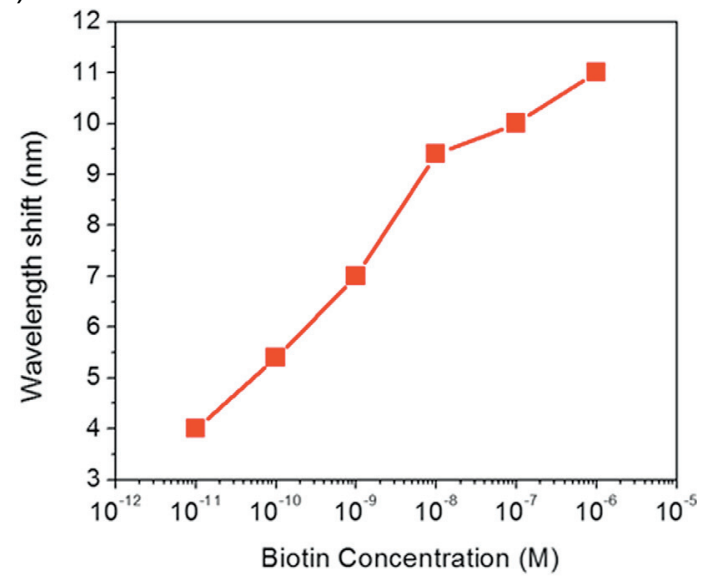

(d)

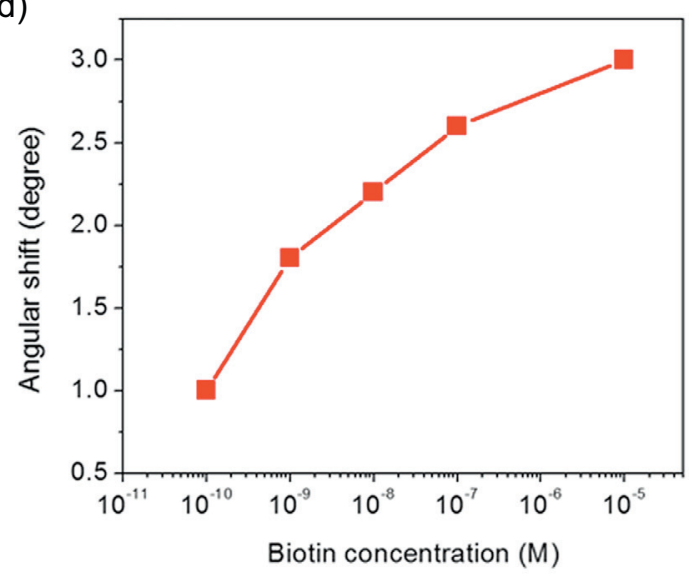

Figure 7. Spectral and angular detection of low molecular-weight biomolecules (biotin): (a) reflectance spectra of the sensor at $30^{\circ}$ angle of incidence for different concentrations of biotin in PBS, (b) the variation of wavelength shift with different concentrations of biotin, (c) reflectance spectra of the sensor device at $1250 \mathrm{~nm}$ for different concentrations of biotin in PBS, and (d) the variation of angular shift with different concentrations of biotin.

(a)

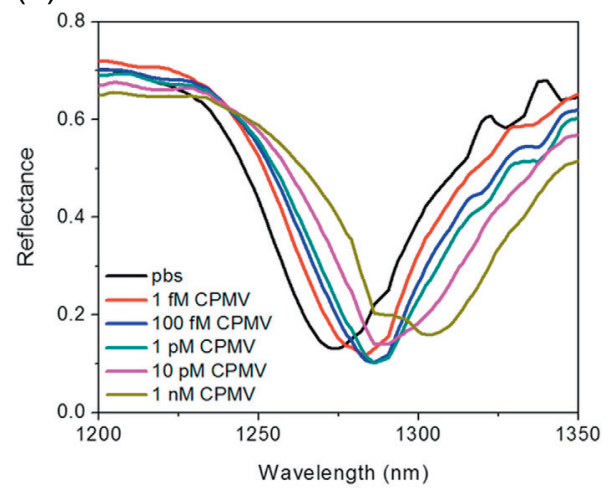

(b)

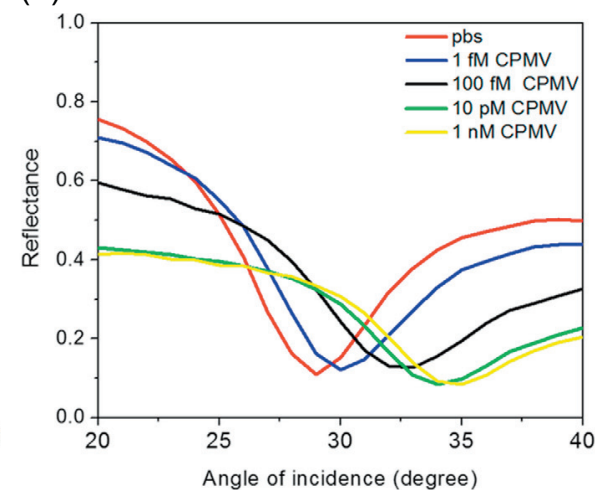

Figure 8. Spectral and angular detection of higher molecular-weight macromolecules (CPMV): (a) reflectance spectra of the sensor at $30^{\circ}$ angle of incidence for different concentrations of CPMV in PBS, (b) reflectance spectra of the sensor device at $1250 \mathrm{~nm}$ for different concentrations of CPMV in PBS.

is around 0.0006 . This sensor device provides different sensitivity from visible to near IR wavelengths, with a maximum spectral and angular sensitivity recorded at $1300 \mathrm{~nm}$, which is around $30,000 \mathrm{~nm} / \mathrm{RIU}$ and $2500^{\circ} / \mathrm{RIU}$, respectively. The minimum values recorded at $530 \mathrm{~nm}$ are $13,333 \mathrm{~nm} / \mathrm{RIU}$ and $2333^{\circ} / \mathrm{RIU}$ for spectral and angular sensitivity, respectively. 
(a)

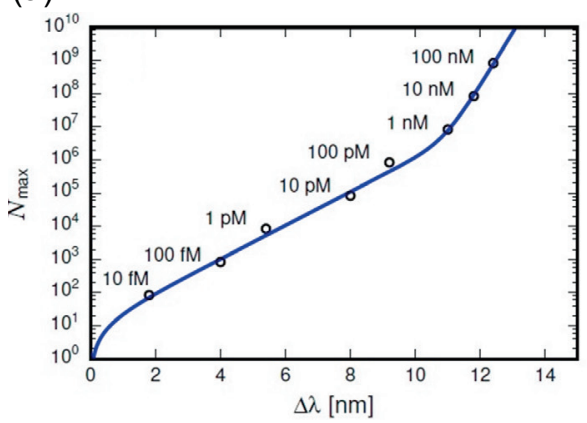

(b)

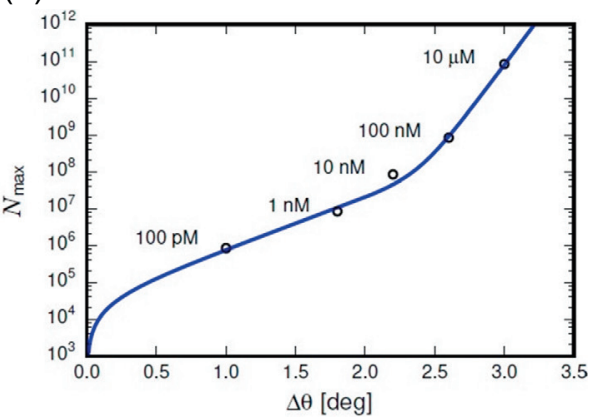

Figure 9. Experimental results for the maximum number of biotin molecules, $N_{\max }$, adsorbed in the illuminated sensor areas in the long-time limit for the mode located at $1280 \mathrm{~nm}$, (a) versus the corresponding wavelength shift $\Delta \lambda(c)$ and (b) versus the corresponding angular shift $\Delta \theta(c)$.

Since the sensor device shows different sensitivities for each mode, there is flexibility in the selection of a particular mode for identification of specific biomolecules. In other words, there is the option of using a lower sensitivity mode for the detection of higher molecular weight biomolecules and a higher sensitivity mode for the detection of lower molecular weight biomolecules.

\section{Biosensing experiments}

In the biosensing experiments based on spectral and angular interrogation schemes, we only consider the mode at $1300 \mathrm{~nm}$ because it provides enhanced sensitivity.

\subsection{Lower molecular-weight biomolecule detection}

To demonstrate the capabilities of the sensor device for the detection of lower molecular-weight biomolecules $(<500 \mathrm{Da})$, both spectral and angular interrogation schemes were used for sensing biotin (molecular weight $=244 \mathrm{Da}$ ). The importance of the detection of biotin is that it is a model system for small molecule compounds such as other vitamins, cancer-specific proteins, hormones, therapeutics, or contaminants such as pesticides or toxins. Importantly, circulating tumor cells overexpress small proteins $(<500 \mathrm{Da})$ very early that are extremely difficult to detect with currently available sensing technologies. The well-known streptavidin-biotin affinity model was used for the capture of biotin [13]. The measurement of refractive index change caused by the capture of biotin at the sensor surface is possible using this set up. By using a single injection procedure (in batch mode), the performance of the sensor was monitored based on the resonant wavelength and angular shift in the reflectance spectra when different concentrations ( $100 \mathrm{pM}$ to $10 \mu \mathrm{M})$ of biotin in phosphate buffered saline (PBS) were injected into the sensor microchannel. Note that the reflectance spectra of the sensor were recorded after a reaction time of $40 \mathrm{~min}$ and PBS was introduced into the microchannel to remove the unbound and weakly-attached biotin molecules before each injection of a new concentration of biotin. The responses of the device during the detection of different concentrations of biotin in spectral and angular scans are shown in Figures $7 \mathrm{a}$ and $7 \mathrm{c}$, respectively. A red shift in wavelength and positive angular shift were obtained when the biotin concentration was increased, which is due to the increase in refractive index by the capture of biomolecules. In both scans a nonlinear variation of the response as a function of the biotin concentration was observed (Figures $7 \mathrm{~b}$ and $7 \mathrm{~d}$ ).

\subsection{Higher molecular-weight macromolecule detection}

We then performed experiments in the absence of surface functionalization, for detecting high molecular weight macromolecules such as Cowpea mosaic virus (CPMV, molecular weight $=5.6 \times 10^{6} \mathrm{Da}$ ), to demonstrate the extraordinary flexibility of the sensor device. We followed the method described in reference [22] for the preparation of CPMV. The CPMV is a plant virus that we choose as a safe model system to mimic infectious disease. Different concentrations ( $1 \mathrm{fM}$ to $1 \mathrm{nM}$ ) of CPMV were prepared in PBS and then injected into the sensor microchannel. The reflectance spectra of the sensor with highly diluted CPMV solutions were recorded after a reaction time of $20 \mathrm{~min}$ and PBS was injected into the channel to remove unbound and weakly-attached CPMV. Since the molecular weight of CPMV is huge, a large wavelength shift (Figure 8a) and angular shift (Figure 8b) even for $1 \mathrm{fM}$ CPMV was obtained and the sensor saturates very quickly. Specificity to detect viruses in complex media (blood or other body fluids and tissue samples) needs to be achieved for commercial implementation, and will be a future direction.

\subsection{Quantification of adsorbed molecules on the sensor surface}

The sensitivity of the wavelength and angular shift to the number of adsorbed molecules on the sensor surface is analyzed in this section. We consider the saturation values of the wavelength $(\Delta \lambda)$ and angular $(\Delta \theta)$ shift (which occurs approximately after $45 \mathrm{~min}$ ) for different concentrations $c$ of biotin in PBS. The shift of the resonance wavelength and angle depend on the number of bound molecules $N(c)$ in the sensing 
region. Though we cannot directly measure the precise value of $N(c)$, we can reliably estimate an upper bound $N_{\max }(c)$ based on the sensor parameters, such that the actual $N(c) \leq N_{\max }(c)$ at any concentration. The result is that $N_{\max }(c)=8.4 c \times 10^{15} \mathrm{M}^{-1}$. We observed in references $[13,14]$ that there was a nonlinear relationship between $N_{\max }(c)$ and $\Delta \lambda(c)$, and $N_{\max }(c)$ and $\Delta \theta(c)$, the shift in resonance wavelength and angle at concentration $c$. This behavior could be accurately represented using a phenomenological double-exponential fitting function, and we find an analogous functional relationship between $N_{\max }(c)$ and $\Delta \theta(c)$, and $N_{\max }(c)$ and $\Delta \lambda(c)$. It takes the form:

$$
\begin{aligned}
& N_{\max }=A_{1}\left(e^{\Delta \lambda / \beta_{1}}-1\right)+A_{2}\left(e^{\Delta \lambda / \beta_{2}}-1\right), \\
& N_{\max }=A_{1}\left(e^{\Delta \theta / \beta_{1}}-1\right)+A_{2}\left(e^{\Delta \theta / \beta_{2}}-1\right),
\end{aligned}
$$

where $A_{1}, A_{2}, \beta_{1}, \beta_{2}$, are fitting parameters. The function is chosen such that the limiting behavior at small $\Delta \lambda$ and $\Delta \theta$ is linear, as we would expect in the case of very few adsorbed particles. Figure 9 shows the experimental $N_{\max }$ versus $\Delta \lambda$ and $\Delta \theta$ results for biotin, with the best-fits of equations (1) and (2) drawn as solid curves. In the case of CPMV, at the smallest probed concentration $(1 \mathrm{fM})$ the value for $N_{\max }$ is approximately eight adsorbed particles, indicating that we are close to the single-particle detection regime.

\section{Summary and outlook}

In summary, we showed that HMM offers an extremely sensitive transducer for optical label free biosensing. We have demonstrated the excitation of high-k BPP modes in HMMs using a metallic grating coupler, thus overcoming the bulkiness problem of exciting SPPs using the Kretschmann configuration and allowing for multimodal excitation of several BPP modes that have their momentum matched due to higher order grating modes.

The high-k BPP modes exhibited extreme sensitivity for both angular and spectral sensitivities reaching $30,000 \mathrm{~nm} / \mathrm{RIU}$ and $2500^{\circ} / \mathrm{RIU}$ respectively, allowing for detection of low molecular weight biomolecules at low concentration. The multimodal differential response of our sensor offers a promising opportunity to design an assay for the selective detection of higher- and lower-molecular-weight biomolecules in highly diluted solutions. Both miniaturization and multiplexing are two important features for new generation lab-on-a-chip sensors. The embedding of the HMM photonic chips in biocompatible matrices (e.g., silk) could also allow for transcutaneous implantation. The multiplexing assay is very important in sensing applications because it brings specificity properties to ligand-free sensors and adds specificity features to ligand-functionalized sensors. The integration of a metamaterial-based photonic chip with micrometer-sized channels can be adapted to allow microfluidic manipulation of real biological samples, including biochemical operations, amplification by polymerase chain reaction, filtration and blood separation. Furthermore, the biosensing platform can be employed for a wide range of molecule concentrations: from highly diluted concentrations (10 fM) to the more concentrated multi-analyte samples $(10 \mu \mathrm{M})$. In future, efforts will be dedicated to verify the ability of the multimodes to distinguish between parasitic molecules and targeted biomolecules by optimizing specific biomolecular interactions and by individuating highly selective bio-receptors. In addition, in order to fully realize advanced POC tools, the fabrication costs are still a relevant issue. Lithographically free nanophotonic platforms would provide a valid solution for this problem. Not only would the fabrication costs be dramatically reduced, but also the sensing area could be significantly enlarged, allowing for a multi-channel sensing platform.

Acknowledgements. We acknowledge support from the Ohio Third Frontier Project "Research Cluster on Surfaces in Advanced Materials (RC-SAM) at Case Western Reserve University". This work was also supported in part by Grant \# 2013126 from the Doris Duke Charitable Foundation. In addition, we acknowledge the support of the MORE Center at Case Western Reserve University and the GU malignancies program of the Case Comprehensive Cancer Center.

\section{References}

1. S.M. Borisoy, O.S. Wolfbeis, Chem. Rev. 108 (2008) 423.

2. J.N. Anker et al., Nat. Mater. 7 (2008) 442.

3. A.G. Brolo, Nat. Photon. 6 (2012) 709.

4. S. Zeng et al., Chem. Soc. Rev. 43 (2014) 3426.

5. P. Zijlstra, P.M.R. Paulo, M. Orrit, Nat. Nanotech. 7 (2012) 379.

6. J. Homola, S.S. Yee, G. Gauglitz, Sens. Actuators B 54 (1999) 3.

7. S. Zeng et al., Adv. Mater. 27 (2015) 6163.

8. H. Chen et al., Langmuir 24 (2008) 5233.

9. T. Sannomiya, J. Voros, Trends Biotechnol. 29 (2011) 343.

10. K.M. Mayer et al., Nanotechnology 21 (2010) 255503.

11. M. Svedendahl, R. Verre, M. Kall, Light: Science \& Applications 3 (2014) 01.

12. A.V. Kabashin et al., Nat. Mater. 8 (2009) 867.

13. K.V. Sreekanth et al., Nat. Mater. 15 (2016) 621.

14. K.V. Sreekanth et al., Adv. Opt. Mater. (2016) DOI: 10.1002/ adom.201600448.

15. A. Poddubny et al., Nat. Photon. 7 (2013) 948.

16. I. Avrutsky et al., Phys. Rev. B 75 (2007) 241402.

17. K.V. Sreekanth, A. De Luca, G. Strangi, Sci. Rep. 3 (2013) 3291.

18. K.V. Sreekanth et al., Sci. Rep. 4 (2014) 6340.

19. K.V. Sreekanth, A. De Luca, G. Strangi, J. Opt. 16 (2014) 105103.

20. C.L. Cortes et al., J. Opt. 14 (2012) 063001.

21. K.V. Sreekanth et al., Sci. Rep. 6 (2016) 26272.

22. A.M. Wen et al., Bioconjug. Chem. 26 (2015) 51. 